\title{
Microwave data refine picture of Universe
}

Youprobably wouldn'texpect measuring the Universe to be easy. But turning data from the Wilkinson Microwave Anisotropy Probe (WMAP) into a map of the Big Bang's afterglow has been an all-consuming task.

"We just had toget this out to get on with ourlives," says Lyman Page of PrincetonUniversity, New Jersey. Page and his colleagues have spent the past three years analysing the latestdata from WMAP, a satellite launched in 2001 to study patterns

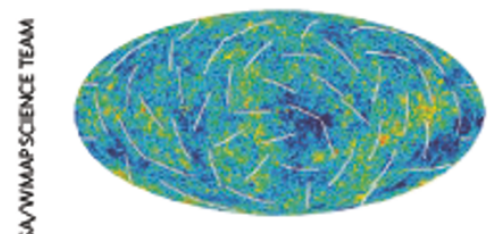

Hotspots: afterglow of the Big Bang. in the radiation known as the cosmic microwave background. These patterns, which reflect the state of the Universe at the time the first atoms formed, hold clues to its structure, history and composition.

The team's results, revealed on 16 March, broadly confirmits first conclusions - presented in 2003 after analysis of a single year of data.

The 2003 data provided powerful support for the idea that the Universe was dominated by things not directly observable - dark matter and dark energy. To improve on these results, which were based onmaps of temperature fluctuations, the team mapped the polarization of the microwaves. The temperature signals are tiny (between 30 and 70 millionths of a kelvin); the polarization signal is 100 times fainter still. To detect the polariz ation signal meant characterizing completely the noise in the instrument. "At times you just thought, oh my gosh, how are we ever going to get this right?" says Gary Hinshaw, who coordinated the analysis.

For example, an amplifier on the satellite introduces complex noise into the data by contaminating each observation with a lingering trace of its previous measurement. The team had to find a way to correct for this across the 3 million pixels of the full-sky map. Often, they would try an approach only to find that it introducednew artefacts.

"It was just gruesome detail day after day," says Charles Bennett, WMAP's principal investigator.

From the polarization maps, the team precisely calculated the Universe's 'optical depth', one of the six fundamental properties of the cosmos. This allowedit to estimate that the firststars formed $\mathbf{4 0 0}$ millionyears after the Big Bang.

It calculated another basic property, know as the inflation parameter, to be 0.95 with an uncertainty of less than 0.02 . Theories of inflation, which hold that the Universe had a dramatic growth spurt in its firstmoments, predict that the inflation parameter should not be equal to one.

The results, submitted to The Astrophysical Joumal, were released four days before the WMAP team was due to submit a request for further funding to NASA. The work goes ever on. Jenny Hogan 